\title{
THE CLOSEST PACKING OF SPHERICAL CAPS IN $n$ DIMENSIONS
}

\author{
by R. A. RANKIN
}

(Received lst December, 1954)

1. Let $S_{n}$ denote the "surface" of an $n$-dimensional unit sphere in Euclidean space of $n$ dimensions. We may suppose that the sphere is centred at the origin of coordinates $O$, so that the points $P\left(x_{1}, x_{2}, \ldots, x_{n}\right)$ of $S_{n}$ satisfy

$$
x_{1}^{2}+x_{2}^{2}+\ldots+x_{n}^{2}=1 \text {. }
$$

We suppose that $n \geqslant 2$.

By a spherical cap $C(\alpha)$ of angular radius $\alpha \leqslant \pi$ and centre $P$ on $S_{n}$, we mean the set of points $Q$ of $S_{n}$ for which the angle $Q O P$ is less than or equal to $\alpha$. Our object is to obtain an upper bound for the maximum number $N(\alpha)$ of non-overlapping caps $C(\alpha)$ which can be placed on $S_{n}$, and to investigate this upper bound for large values of $n$.

It is clear that $N(\alpha)$ is a decreasing function of $\alpha$, and that $N(\alpha)$ is continuous on the left for all $\alpha$ in the interval $0<\alpha \leqslant \pi$. We prove the following theorems :

Theorem 1. We have

(i) $N(\alpha)=1$ for $\frac{1}{2} \pi<\alpha \leqslant \pi$;

(ii) $N(\alpha)=\left[\frac{2 \sin ^{2} \alpha}{2 \sin ^{2} \alpha-1}\right]$ for $\frac{1}{4} \pi+\frac{1}{2} \sin ^{-1} \frac{1}{n} \leqslant \alpha \leqslant \frac{1}{2} \pi$;

(iii) $N(\alpha)=n+1$ for $\frac{1}{4} \pi<\alpha \leqslant \frac{1}{4} \pi+\frac{1}{2} \sin ^{-1} \frac{1}{n}$;

(iv) $N\left(\frac{1}{4} \pi\right)=2 n$.

Theorem 2. If $0<\alpha<\frac{1}{4} \pi$ and $\beta=\sin ^{-1}(\sqrt{ } 2 \sin \alpha)$, then

$$
N(\alpha) \leqslant \frac{\pi^{k} \Gamma\left(\frac{n-1}{2}\right) \sin \beta \tan \beta}{2 \Gamma\left(\frac{n}{2}\right) \int_{0}^{\beta}(\sin \theta)^{n-2}(\cos \theta-\cos \beta) d \theta}=N^{*}(\alpha),
$$

say. Further, for large $n$,

provided that $\sec 2 \alpha=o(n)$.

$$
N^{*}(\alpha) \sim \frac{\left\{\frac{1}{2} \pi n^{3} \cos 2 \alpha\right\}^{t}}{(\sqrt{ } 2 \sin \alpha)^{n-1}},
$$

We note that it follows immediately from Theorem 1 that if $n+2$ spherical caps can be placed on $S_{n}$, then so can $2 n$. This is a generalisation of the corresponding result for $n=3$ obtained by Schütte and van der Waerden (4). As Professor Rogers has since pointed out to me, this result has already been obtained by Hajós and Davenport (see (2), p. 188).

Theorem 2 is proved by the Blichfeldt density method. See, for example, (3).

An application to the theory of positive definite quadratic forms is given in $\S 4$.

2. Proof of 'Theorem 1. We shall say that $m$ points $P_{1}, P_{2}, \ldots, P_{m}$ on $S_{n}$ form an $\alpha$-packing, for $0<\alpha \leqslant \frac{1}{2} \pi$, if the angle subtended by any two at $O$ is not less than $2 \alpha$. Thus, if $P_{\text {v }}$ has coordinates $\left(x_{v 1}, x_{\nu 2}, \ldots, x_{v n}\right)$ we have

$$
\alpha_{\mu \nu}=\sum_{k=1}^{n} x_{\mu k} x_{\nu k} \leqslant \cos 2 \alpha \quad(\mu \neq \nu) .
$$


If a cap $C(\alpha)$ is centred at each point $P_{v}$, it is clear that no two caps will overlap. The inequality (4) is equivalent to

$$
r_{\mu \nu} \geqslant 2 \sin \alpha=\rho \quad(\mu \neq \nu)
$$

say, where $r_{\mu \nu}$ is the linear distance between $P_{\mu}$ and $P_{\nu}$.

LEMMA 1. If the points $P_{1}, P_{2}, \ldots, P_{m}$ form an $\alpha$-packing on $S_{n}$, and if $P$ is any point on $S_{n}$, then

$$
\left(\sum_{\nu=1}^{m} r_{v}^{2}\right)^{2}-4 m \sum_{v=1}^{m} r_{\nu}^{2}+2 m(m-1) \rho^{2} \leqslant 0
$$

where $r_{v}$ is the linear distance from $P$ to $P_{v}$.

Proof. We may suppose, without loss of generality, that $P$ is the point $(1,0,0, \ldots, 0)$. Then, by (5), we have

Since

$$
\begin{aligned}
\frac{1}{2} m(m-1) \rho^{2} & \leqslant \sum_{k=1}^{n} \underset{1 \leqslant \mu<\nu \leqslant m}{\sum}\left(x_{\mu k}-x_{\nu k}\right)^{2} \\
& =\sum_{k=1}^{n}\left\{m \sum_{\nu=1}^{m} x_{\nu k}^{2}-\left(\sum_{\nu=1}^{m} x_{\nu k}\right)^{2}\right\} \\
& =m \sum_{\nu=1}^{m}\left(1-x_{\nu 1}\right)^{2}-\left\{m-\sum_{\nu=1}^{m} x_{\nu 1}\right\}^{2}+\sum_{k=2}^{n}\left\{m \sum_{\nu=1}^{m} x_{\nu k}^{2}-\left(\sum_{\nu=1}^{m} x_{\nu k}\right)^{2}\right\} .
\end{aligned}
$$

we deduce that

$$
r_{v}^{2}=\left(1-x_{v 1}\right)^{2}+x_{v 2}^{2}+\ldots+x_{v n}^{2}=2\left(1-x_{v 1}\right)
$$

and the lemma follows.

$$
\frac{1}{2} m(m-1) \rho^{2} \leqslant m \sum_{\nu=1}^{m} r_{\nu}^{2}-\frac{1}{4}\left(\sum_{\nu=1}^{m} r_{\nu}^{2}\right)^{2}-\sum_{k=2}^{n}\left(\sum_{\nu=1}^{m} x_{\nu k}\right)^{2},
$$

LEMMA 2. If $\frac{1}{4} \pi<\alpha \leqslant \frac{1}{2} \pi$, then

$$
N(\alpha) \leqslant\left[\frac{2 \sin ^{2} \alpha}{2 \sin ^{2} \alpha-1}\right] .
$$

Proof. Suppose that $m$ non-overlapping caps $C(\alpha)$ can be placed on $S_{n}$. Then, for any point $P$ on $S_{n}$, we have, by Lemma 1 ,

$$
\left\{\sum_{\nu=1}^{m} r_{\nu}^{2}-2 m\right\}^{2} \leqslant 4 m^{2}-2 m(m-1) \rho^{2}=4 m^{2}\left\{1-2\left(1-\frac{1}{m}\right) \sin ^{2} \alpha\right\} \text {. }
$$

It follows that

$$
\begin{array}{ll} 
& 2\left(1-\frac{1}{m}\right) \sin ^{2} \alpha \leqslant 1 ; \\
\text { i.e., } \quad \frac{2}{m} \sin ^{2} \alpha \geqslant 2 \sin ^{2} \alpha-1,
\end{array}
$$

which proves the result stated.

LEMMA 3. If $\alpha_{k}=\frac{1}{4} \pi+\frac{1}{2} \sin ^{-1} \frac{1}{k}(l \leqslant k \leqslant n)$, then

Proof. Since

$$
N\left(\alpha_{k}\right)=k+1 \text {. }
$$

$$
\frac{2 \sin ^{2} \alpha_{k}}{2 \sin ^{2} \alpha_{k}-1}=k+1
$$


we only have to prove that $k+1$ caps $C\left(\alpha_{k}\right)$ can be placed on $S_{n}$. It is convenient to suppose that $S_{n}$ is the intersection of $S_{n+1}$ with the hyperplane

$$
x_{1}+x_{2}+\ldots+x_{n+1}=0 \text {. }
$$

Choose points $P_{1}, P_{2}, \ldots, P_{k+1}$ on $S_{n}$ as follows. For $P_{v}$ we take

$$
x_{\nu j}=-\frac{\lambda}{k+1} \quad(j \neq \nu, j \leqslant k+1), \quad x_{\nu v}=\lambda-\frac{\lambda}{k+1}, \quad x_{\nu j}=0 \quad(j>k+1),
$$

where

$$
\lambda=\sqrt{\left(\frac{k+1}{k}\right)} \text {. }
$$

It is easily verified that the angle $P_{\mu} O P_{\nu}$ is $2 \alpha_{k}$ for all $\mu \neq \nu$. Hence a cap $C\left(\alpha_{k}\right)$ can be centred at each of the $k+1$ points without overlapping.

Since $N(\alpha)$ and $2 \sin ^{2} \alpha /\left(2 \sin ^{2} \alpha-1\right)$ are decreasing functions of $\alpha$, for $\alpha>\frac{1}{4} \pi$, part (ii) of Theorem 1 follows immediately from Lemmas 2 and 3 . Since (i) is trivial, it remains to prove (iii) and (iv).

We observe first that

$$
N\left(\frac{1}{4} \pi\right) \geqslant 2 n,
$$

since we may take $2 n$ non-overlapping caps $C\left(\frac{1}{4} \pi\right)$ centred at the points $( \pm 1,0,0, \ldots, 0)$, $(0, \pm 1,0, \ldots, 0), \ldots,(0,0, \ldots, 0, \pm 1)$. Note that $2 n \geqslant n+2$.

We prove (iii) by induction. It is true for $n=2$; we assume its truth for packings on $S_{n-1}$, and deduce the corresponding result for $S_{n}$.

Suppose that, for some $\alpha$ satisfying $\frac{1}{4} \pi \leqslant \alpha \leqslant \alpha_{n}$, there exists an $\alpha$-packing of $n+2$ points $P_{1}, P_{2}, \ldots, P_{n+2}$ on $S_{n}$, where $S_{n}$ is given by (1). We prove that $\alpha=1 \pi$. For suppose not, so that $\frac{1}{4} \pi<\alpha<\alpha_{n}$.

Without loss of generality we may suppose that $P_{n+2}$ is the point $(0,0, \ldots, 0,-1)$. Then, by (2),

Hence

$$
\alpha_{\mu \nu}=\sum_{k=1}^{n} x_{\mu k} x_{\nu k} \leqslant \cos 2 \alpha<0 \quad(\mu \neq \nu) .
$$

None of the points $P_{1}, P_{2}, \ldots, P_{n+1}$ can be $(0,0, \ldots, 0,1)$; for if $P_{n+1}=(0,0, \ldots, 0,1)$ we should have, by (7),

$$
x_{\mu n}=\alpha_{\mu, n+1} \leqslant \cos 2 \alpha<0 \quad(\mu \leqslant n),
$$

which contradicts (8). Hence $0<x_{\mu n}<1$ for $\mu \leqslant n+1$.

Write

so that

$$
\lambda_{\mu}=\sqrt{ }\left(x_{\mu 1}^{2}+x_{\mu 2}^{2}+\ldots+x_{\mu, n-1}^{2}\right)=\sqrt{ }\left(1-x_{\mu n}^{2}\right) \quad(\mu \leqslant n+1),
$$

Now consider the $n+l$ points

$$
0<\lambda_{\mu} \leqslant \sqrt{ }\left(1-\cos ^{2} 2 \alpha\right) \text {. }
$$

$$
P_{\mu}^{\prime}=\left(\frac{x_{\mu_{1}}}{\lambda_{\mu}}, \frac{x_{\mu 2}}{\lambda_{\mu}}, \ldots, \frac{x_{\mu, n-1}}{\lambda_{\mu}}, 0\right)
$$

where $\mu \leqslant n+1$; they lie on an $S_{n-1}$, namely

We have, for $\mu \neq \nu$, by (7), (8) and (9),

$$
x_{1}^{2}+x_{2}^{2}+\ldots+x_{n-1}^{2}=1 \text {. }
$$

$$
\alpha_{\mu \nu}^{\prime}=\cos P_{\mu}^{\prime} O P_{\nu}^{\prime}=\frac{1}{\lambda_{\mu} \lambda_{\nu}}\left(\alpha_{\mu \nu}-x_{\mu n} x_{\nu n}\right) \leqslant \frac{1}{\lambda_{\mu} \lambda_{\nu}}\left(\cos 2 \alpha-\cos ^{2} 2 \alpha\right) \leqslant \frac{\cos 2 \alpha}{1+\cos 2 \alpha}=\gamma,
$$


say. Now $-1<\gamma<0$, since $\alpha<\frac{1}{3} \pi$, and hence, for some $\psi$ satisfying

$$
\frac{1}{4} \pi<\psi<\frac{1}{2} \pi
$$

we have

$$
\alpha_{\mu \nu}^{\prime} \leqslant \gamma=\cos 2 \psi
$$

Hence we can place $n+1$ non-overlapping caps $C(\psi)$ on $S_{n-1}$, and therefore, by the induction hypothesis, $\psi \leqslant \frac{1}{4} \pi$, which contradicts (12). Accordingly we must have $\alpha=\frac{1}{4} \pi$, and this proves (iii).

Part (iv) will follow by (6) if we show that there does not exist a $\frac{1}{4} \pi$-packing of $2 n+1$ points on $S_{n}$. This is true for $n=2$ and, as before, we assume its truth for $S_{n-1}$. Suppose therefore that such a $\frac{1}{4} \pi$-packing exists on $S_{n}$. Take $P_{2 n+1}$ to be $(0,0, \ldots, 0,-1)$. Then, as before, we have

$$
x_{\mu n}=-\alpha_{\mu, 2 n+1} \geqslant 0 \quad(\mu \leqslant 2 n) .
$$

At most one of the points $P_{1}, P_{2}, \ldots, P_{2 n}$ can be $(0,0, \ldots, 0,1)$, so that we can assume that $\left|x_{\mu n}\right|<1$ for $\mu \leqslant 2 n-1$. Define $2 n-1$ new points $P_{\mu}^{\prime}$ as in (10) for $\mu \leqslant 2 n-1$, where $\lambda_{\mu}$ is given by (9) and $\lambda_{\mu}>0$. These $2 n-1$ points lie on the $S_{n-1}(11)$, and we have

$$
\alpha_{\mu \nu}^{\prime}=\cos P_{\mu}^{\prime} O P_{\nu}^{\prime}=\frac{1}{\lambda_{\mu} \lambda_{\nu}}\left(\alpha_{\mu \nu}-x_{\mu n} x_{\nu n}\right) \leqslant 0
$$

for $1 \leqslant \mu<\nu \leqslant 2 n-1$. Thus $P_{1}^{\prime}, P_{2}^{\prime}, \ldots, P_{2 n-1}^{\prime}$ form a $\frac{1}{4} \pi$-packing on $S_{n-1}$ and this contradicts our induction hypothesis. It follows that part (iv) of the theorem is true for all $n \geqslant 2$.

It may be noted that the above method of proof also shows that, in every $\frac{1}{4} \pi$-packing of $2 n$ points on $S_{n}$, the coordinates may be chosen so that the $2 n$ points take the form

$$
( \pm 1,0, \ldots, 0), \ldots,(0,0, \ldots, 0, \pm 1) \text {. }
$$

3. Proof of Theorem 2. We first prove the

Lemma 4. If $0<\beta<\frac{1}{2} \pi$ and if $\tan \beta=o(\sqrt{ } n)$ for large $n$, then

$$
\int_{0}^{\beta}(\sin \theta)^{n-2}(\cos \theta-\cos \beta) d \theta \sim \frac{\sec ^{2} \beta}{n^{2}}(\sin \beta)^{n+1} .
$$

Proof. We have, putting $t=(n-1) \log (\sin \beta / \sin \theta)$,

$$
\cos \beta \int_{0}^{\beta}(\sin \theta)^{n-2} d \theta=\frac{(\sin \beta)^{n-1}}{n-1} \int_{0}^{\infty} \frac{e^{-t} d t}{\left\{1+\tan ^{2} \beta\left(1-e^{-2 t /(n-1)}\right)\right\}}
$$

Now, by applying Taylor's theorem to

$$
f(x)=(1+x)^{-\frac{1}{1}}-1+\frac{1}{2} x,
$$

we easily see that $0 \leqslant f(x) \leqslant \frac{3}{8} x^{2}$ for all $x \geqslant 0$, and accordingly we have, for some $\vartheta_{t}$ satisfying $0 \leqslant \vartheta_{t} \leqslant 1$,

$$
\begin{aligned}
\cos \beta \int_{0}^{\beta}(\sin \theta)^{n-2} d \theta & =\frac{(\sin \beta)^{n-1}}{n-1} \int_{0}^{\infty} e^{-t}\left\{1-\frac{1}{2} \tan ^{2} \beta\left(1-e^{-2 t /(n-1)}\right)+\frac{5}{8} \vartheta_{t} \tan ^{4} \beta\left(1-e^{-2 t /(n-1)}\right)^{2}\right\} d t \\
& =\frac{(\sin \beta)^{n-1}}{n-1}\left\{1-\frac{\tan ^{2} \beta}{n+1}+\frac{3 \vartheta \tan ^{4} \beta}{(n+1)(n+3)}\right\},
\end{aligned}
$$

where $0 \leqslant \vartheta \leqslant 1$. From this the lemma follows easily.

Suppose now that there are $N$ non-overlapping caps $C(\alpha)$ on $S_{n}$, where $\alpha<\frac{\pi}{4}$. We replace each cap by a concentric $\operatorname{cap} C(\beta)$ where

$$
\beta=\sin ^{-1}(\sqrt{ } 2 \sin \alpha) \text {. }
$$


Further, we attach a density

$$
\sigma(r)=\frac{\cos \beta}{\sin ^{2} \beta}\left(R^{2}-r^{2}\right)=\frac{2 \cos \beta}{\sin ^{2} \beta}(\cos \theta-\cos \beta)
$$

to points $Q$ on $C(\beta)$ at a distance $r=2 \sin \frac{1}{2} \theta$ from the centre of $C(\beta)$. Here $R=2 \sin \frac{1}{2} \beta$ and is the linear radius of $C(\beta)$. The content of a unit sphere in $n$-dimensions is

and the total "mass" of $C(\beta)$ is

$$
J_{n}=\frac{\pi^{\ddagger n}}{\Gamma\left(\frac{n}{2}+1\right)}
$$

$$
\begin{aligned}
M & =\int_{0}^{\beta}(n-1) J_{n-1}(\sin \theta)^{n-2} \sigma\left(2 \sin \frac{1}{2} \theta\right) d \theta \\
& =2(n-1) \frac{\cos \beta}{\sin ^{2} \beta} J_{n-1} \int_{0}^{\beta}(\sin \theta)^{n-2}(\cos \theta-\cos \beta) d \theta .
\end{aligned}
$$

Let $P$ be any point of $S_{n}$. Then either $P$ belongs to no cap $C(\beta)$, in which case the density at $P$ is zero, or else $P$ belongs to $m \geqslant 1$ such caps centred at $P_{1}, P_{2}, \ldots, P_{m}$, say. In the latter case, the total density at $P$ is, in the notation of Lemma 1 ,

Thus

$$
\sigma_{P}=\sum_{\nu=1}^{m} \sigma\left(r_{\nu}\right)=\frac{\cos \beta}{\sin ^{2} \beta}\left\{m R^{2}-\sum_{\nu=1}^{m} r_{\nu}^{2}\right\}
$$

$$
\sum_{\nu=1}^{m} r_{v}^{2}=4 m \sin ^{2} \frac{1}{2} \beta-\sigma_{P} \sin \beta \tan \beta=4 \sin ^{2} \frac{1}{2} \beta\left\{m-\frac{1}{2}(1+\sec \beta) \sigma_{P}\right\}
$$

and so, by Lemma 1,

$$
0 \geqslant 16 \sin ^{4} \frac{1}{2} \beta\left\{m-\frac{1}{2}(1+\sec \beta) \sigma_{P}\right\}^{2}-16 m \sin ^{2} \frac{1}{2} \beta\left\{m-\frac{1}{2}(1+\sec \beta) \sigma_{P}\right\}+4 m(m-1) \sin ^{2} \beta .
$$

This reduces to

$$
4 m\left(1-\sigma_{P}\right) \geqslant \sigma_{P}^{2} \tan ^{2} \beta,
$$

from which it follows that $\sigma_{P} \leqslant 1$.

We deduce that $N M$ is less than the total surface area of $S_{n}$, and so, by (13),

$$
N \leqslant \frac{n J_{n}}{M}=\frac{n J_{n} \sin \beta \tan \beta}{2(n-1) J_{n-1} \int_{0}^{\beta}(\sin \theta)^{n-2}(\cos \theta-\cos \beta) d \theta} .
$$

This proves the first part of Theorem 2, and the second part follows from Lemma 4 .

The "density" of an $\alpha$-packing is

$$
\frac{N \int_{0}^{\alpha}(n-1) J_{n-1}(\sin \theta)^{n-2} d \theta}{n} \overline{J_{n}} \leqslant \frac{\int_{0}^{\alpha}(\sin \theta)^{n-2} d \theta}{\int_{0}^{\beta}(\sin \theta)^{n-2} \sigma\left(2 \sin \frac{1}{2} \theta\right) d \theta}=\rho_{n-1}(\alpha),
$$

say. For small $\alpha$ and fixed $n$ we have

$$
\rho_{n-1}(\alpha) \sim \frac{n+1}{2^{\frac{1}{2}(n+1)}},
$$

the right-hand side being a well-known upper bound for the density of packing of $(n-1)$ dimensional spheres in $(n-1)$-dimensional Euclidean space (3). 
4. By taking $\alpha=\frac{1}{8} \pi$ in $\S 3$ we can get an upper bound for the number $N_{n}$ of unit hyperspheres which can touch a given hypersphere externally without overlapping. We have

say, and

$$
N_{n}=N\left(\frac{1}{6} \pi\right) \leqslant \frac{\pi^{\frac{1}{2}} \Gamma\left(\frac{n-1}{2}\right)}{2 \sqrt{ } 2 \Gamma\left(\frac{n}{2}\right) \int_{0}^{\frac{1}{2} \pi}(\sin \theta)^{n-2}\left(\cos \theta-\cos \frac{1}{4} \pi\right) d \theta}-N_{n}^{*},
$$

for large $n$.

$$
N_{n}^{*} \sim \sqrt{ }\left(\pi n^{3} 2^{n-3}\right)
$$

Consider now a positive definite quadratic form $f\left(u_{1}, u_{2}, \ldots, u_{n}\right)$ in $n$ real variables, and let $m$ be its minimum value for integral $u_{1}, u_{2}, \ldots, u_{n}$, not all zero. Let $m$ be attained by $\mu$ such points $\left(u_{1}, u_{2}, \ldots, u_{n}\right)$. Then it is known (1) that

$$
\mu \leqslant 2^{n+1}-2 \text {. }
$$

We can obtain a better upper bound for $\mu$ for large $n$. For, by a linear transformation of the variables $u_{1}, u_{2}, \ldots, u_{n}, f\left(u_{1}, u_{2}, \ldots, u_{n}\right)$ becomes $x_{1}^{2}+x_{2}^{2}+\ldots+x_{n}^{2}$ and the points at which $m$ is attained become points on the sphere

$$
x_{1}^{2}+x_{2}^{2}+\ldots+x_{n}^{2}=m,
$$

no two of which are at a distance apart of less than $\sqrt{ } m$. Thus

$$
\mu \leqslant N_{n} \leqslant N_{n}^{*} \sim \sqrt{ }\left(\pi n^{3} 2^{n-3}\right)
$$

This is an improvement on (16) for $n>4$. In fact $\left[N_{n}^{*}\right]=60,107,184,309$ for $n=5,6,7,8$, respectively.

It follows from (15) that

$$
\lim _{n \rightarrow \infty} N_{n}^{1 / n} \leqslant \sqrt{ } 2 .
$$

This result has already been obtained by C. A. Rogers in an unpublished manuscript (see (2), p. 188). I observed this after the results of the present paper had been obtained, and I am grateful to Professor Rogers for showing me his method of proof which applies to spherical caps of general radius $\alpha<\frac{1}{4} \pi$. His proof differs from the argument given in $\S 3$ in that the spherical caps of variable density are placed on a smaller concentric sphere of radius $\cos \beta$ and Lemma 1 is used in Blichfeldt's weaker form

$$
\sum_{v=1}^{m} r_{\nu}^{2} \geqslant \frac{1}{2} m(m-1) \rho^{2}
$$

From this an inequality equivalent to (2) is obtained and an upper bound slightly greater than (3) is then deduced.

\section{REFERENCES}

(1) P. Bachmann, Die Arithmetik der quadratischen Formen, Zwoite Abteilung (Berlin, 1923), Chapter 10.

(2) L. Fejes Tóth, Lagerungen in der Ebene auf der Kugel und im Raum (Berlin, 1953).

(3) R. A. Rankin, "On the closest packing of spheres in $n$ dimensions ", Ann. of Math. 48 (1947), 1062-81.

(4) K. Schütte und B. L. van der Waerden, "Auf welcher Kugel haben 5, 6, 7, 8 oder 9 Punkte mit Mindestabstand 1 Platz?" Math. Ann. 123 (1951), 96-124.

\section{The University}

\section{GLaSgow}

